\title{
Criminologie
}

\section{Landreville et les détenu(e)s de par ici}

\section{Lucie Lemonde}

Volume 40, numéro 2, automne 2007

Peines et pénalité au Canada. Autour des travaux de Pierre Landreville

URI : https://id.erudit.org/iderudit/016855ar

DOI : https://doi.org/10.7202/016855ar

Aller au sommaire du numéro

\section{Éditeur(s)}

Les Presses de l'Université de Montréal

ISSN

0316-0041 (imprimé)

1492-1367 (numérique)

Découvrir la revue

Citer cet article

Lemonde, L. (2007). Landreville et les détenu(e)s de par ici. Criminologie, 40(2), 107-125. https://doi.org/10.7202/016855ar

\section{Résumé de l'article}

Pierre Landreville a été une figure marquante du mouvement pour la reconnaissance des droits des détenu(e)s au Québec d'abord, puis au Canada et ailleurs dans le monde. Deux postulats de départ animaient sa recherche dans les prisons québécoises qui a donné lieu en 1976 à la publication de l'ouvrage Les prisons de par ici, à savoir que l'emprisonnement ne se justifie que pour les délinquants qui mettent très sérieusement en danger la collectivité et que les personnes incarcérées conservent tous leurs droits de citoyens, sauf ceux qui leur sont retirés par la loi. L'auteure aborde tour à tour les développements survenus au cours des trente dernières années concernant ces deux postulats. Elle conclut que si les droits des personnes incarcérées sont aujourd'hui législativement reconnus au Canada, le taux d'incarcération y demeure très élevé et la peine d'emprisonnement continue à avoir une fonction d'exclusion des populations les plus marginalisées de la société.
Ce document est protégé par la loi sur le droit d'auteur. L'utilisation des services d’Érudit (y compris la reproduction) est assujettie à sa politique d'utilisation que vous pouvez consulter en ligne.

https://apropos.erudit.org/fr/usagers/politique-dutilisation/ 


\title{
Landreville et les détenu(e)s de par ici
}

\author{
Lucie Lemonde \\ Professeure \\ Département des sciences juridiques, UQÀM \\ lemonde.lucie@uqam.ca
}

RÉSUMÉ - Pierre Landreville a été une figure marquante du mouvement pour la reconnaissance des droits des détenu(e)s au Québec d'abord, puis au Canada et ailleurs dans le monde. Deux postulats de départ animaient sa recherche dans les prisons québécoises qui a donné lieu en 1976 à la publication de l'ouvrage Les prisons de par ici, à savoir que l'emprisonnement ne se justifie que pour les délinquants qui mettent très sérieusement en danger la collectivité et que les personnes incarcérées conservent tous leurs droits de citoyens, sauf ceux qui leur sont retirés par la loi. L'auteure aborde tour à tour les développements survenus au cours des trente dernières années concernant ces deux postulats. Elle conclut que si les droits des personnes incarcérées sont aujourd'hui législativement reconnus au Canada, le taux d'incarcération y demeure très élevé et la peine d'emprisonnement continue à avoir une fonction d'exclusion des populations les plus marginalisées de la société.

ABSTRACT - Pierre Landreville was a key figure of the prisoner's rights movement in Quebec, as well as in Canada and beyond. His seminal work, Les prisons de par ici, which was based on his research in Quebec prisons and published in 1976, was premised on two main postulates. Firstly, that imprisonment is only justified for offenders who pose a real threat to society, and secondly, that a person confined to prison retains all of his civil rights, apart from those expressly or implicitly taken from him by law. The author examines the developments which have occurred during the last three decades with regards to Landreville's postulates. She concludes that while prisoners' rights have now found legislative expression in Canada, the incarceration rate remains high, and imprisonment continues to be an important exclusionary mechanism for the most vulnerable groups of society. 


\section{Introduction}

$\mathrm{Au}$ Canada, le mouvement pour la reconnaissance des droits des personnes incarcérées s'est mis en branle au milieu des années 1970. Un nouveau discours, dont Pierre Landreville est un protagoniste majeur au Québec, fondé non pas uniquement sur l'amélioration des conditions de détention mais sur les droits des détenus comme citoyens, s'articule de façon plus précise au cours de ces années (Landreville et al., 1976; Kaiser, 1971; Pires, 1991; Lemonde, 1995a et 1995b), moment où débute également l'action judiciaire proprement dite. Le mouvement connaît une croissance très rapide et en moins de dix ans, soit de 1975 à 1985, les principales bases des revendications politiques et juridiques sont jetées.

Ce développement spectaculaire suit de peu le mouvement américain. Selon plusieurs analystes, la démocratisation de la société et les luttes en faveur des libertés civiles qui ont transformé la société américaine au cours des années 1950 et 1960 ont eu un impact important sur la naissance du mouvement des droits des détenus (Berkamn, 1979; Huff, 1980; Bronstein, 1980; Jacobs, 1983). Après les Noirs, un groupe marginalisé après l'autre a demandé la redéfinition de ses droits de citoyenneté. Cette extension des libertés civiles s'est par la suite diffusée aux détenus. "The momentum generated by the expansion of civil rights and liberties inexorably penetrated the prison's wall», écrit Jacobs (1983: 24). Dès le début des années 1960, les détenus américains ont assiégé les tribunaux et ont contesté tous les aspects des programmes et pratiques carcérales, ce qui a donné lieu à une véritable révolution légale (Feely et Hanson, 1990).

Sur la scène internationale, les efforts entrepris dès 1926 par la Commission internationale pénale et pénitentiaire (Cornil, 1968) ont abouti en 1955 à l'adoption de l'Ensemble de règles minima pour le traitement des détenus, lors du premier congrès des Nations Unies sur la prévention du crime et le traitement des délinquants (ONU, 1955).

$\mathrm{Au}$ Québec, le premier universitaire à s'être penché sur la question des droits des détenu(e)s est sans contredit Pierre Landreville. Dès le début des années 1970, il publie des articles sur l'application des règles minima des Nations Unies au Canada et, de façon plus générale, sur le fait que les détenus doivent jouir des droits de l'homme au même titre que les autres citoyens (Landreville, 1973; 1974). À ce titre, il est un des pionniers non seulement au Québec mais aussi au Canada où un 
autre universitaire, le professeur Michael Jackson, de l'University of British Columbia (UBC), s'était intéressé à la même époque aux conditions de détention et au processus disciplinaire dans les pénitenciers canadiens (Jackson, 1974).

En 1973, Landreville a mené avec une équipe de chercheurs une enquête sur les conditions de détention et les droits des détenus dans les établissements de détention du Québec. Cette enquête faisait partie d'un projet de recherche de l'Office des droits des détenu(e)s (ODD) financé par la Fondation Doner et a donné lieu à la publication d'un ouvrage important: Les prisons de par ici. L'équipe de chercheurs avait deux objectifs principaux: premièrement, percer le mur du silence et expliquer au public ce qui se passe dans les prisons québécoises et, deuxièmement, proposer une définition concrète des droits des personnes incarcérées.

Je ferai ici deux remarques. Parler en termes de droits des personnes incarcérées était pour l'époque assez avant-gardiste puisque jusqu'alors les revendications pour un régime carcéral juste étaient basées sur des considérations religieuses et humanitaires et n'étaient pas formulées en termes de droits fondamentaux (Jacobs, 1983).

Pour ce qui est de percer le mur du silence, la recherche de Landreville et celles du professeur Jackson ont effectivement eu un effet d'entrấnement et les prisons et les pénitenciers sont devenus des lieux plus ouverts. La première fois que je suis entrée dans un pénitencier, c'était en 1979. De concert avec l'Aide juridique, l'ODD avait initié une cause pour faire déclarer que les conditions de détention dans un pénitencier à sécurité super-maximale, l'Unité spéciale de détention du Centre de développement correctionnel, constituaient un traitement cruel et inusité. Je devais interviewer les prisonniers sur les effets psychologiques et physiques de leurs conditions de détention et de la privation sensorielle. Les entrevues se sont déroulées dans des conditions de très haute suspicion et de très haute sécurité, sans aucun respect du secret professionnel au point que j'ai dû porter plainte au Barreau. Quinze ans plus tard, j'ai pu entrer dans plusieurs pénitenciers et procéder à une recherche pour ma thèse ${ }^{1}$, ce qui est déjà un pas énorme par rapport au passé. Cette fois, non seulement ai-je pu assister aux audiences disciplinaires, mais aussi consulter les dossiers, manger à la cafétéria des employés et rencontrer les détenus directement dans les ailes de détention.

1. Cette thèse intitulée L'émergence et l'impact en droit carcéral était dirigée par Hélène Dumont et Pierre Landreville. Ce dernier a formé et dirigé plusieurs chercheurs dans ce domaine. 
Cette plus grande ouverture, cette nouvelle culture de transparence, qui est aussi le fruit de la lutte des prisonniers et de l'activisme judiciaire, s'est étendue aux processus décisionnels, à l'augmentation de l'information transmise aux détenus et aussi, à la libéralisation du régime des visites contact avec les proches.

Deux postulats de départ animaient la recherche de Landreville en 1973, soit 1) que l'emprisonnement ne se justifie que pour les délinquants qui mettent très sérieusement en danger la collectivité, et 2) que les personnes incarcérées conservent tous leurs droits de citoyens, sauf ceux qui leur sont expressément ou nécessairement retirés par la loi. Pour Landreville, pour que ce principe ne reste pas lettre morte, il doit être inscrit dans la loi.

Dans les deux prochaines sections, j'aborderai tour à tour quelques développements survenus au cours des trente dernières années concernant ces deux postulats, soit la reconnaissance législative des droits des détenu(e)s et, en second lieu, la composition de la population carcérale.

\section{L'évolution de la reconnaissance des droits des détenu(e)s}

Le principe à l'effet que les détenus conservent leurs droits fondamentaux de citoyens, formulé en 1972 par un organisme américain, le National Council on Crime and Delinquency (National Council, 1972) commence peu à peu à faire partie du discours politique au Canada (Canada, 1977a) et est le point de référence fondamental dans l'élaboration du droit carcéral au Canada.

En 1976, à la suite de l'étude exhaustive du professeur Jackson sur le processus disciplinaire dans les pénitenciers canadiens (Jackson, 1974) et à la suite d'une série de soulèvements et de prises d'otages dont les émeutes déclenchées simultanément à l'automne 1976 aux pénitenciers de Saint-Vincent-de-Paul au Québec, de Millhaven en Ontario et celui de la Colombie-Britannique, le gouvernement confie au Sous-comité sur le régime des institutions pénitentiaires au Canada le mandat de faire enquête et rapport sur la situation dans les pénitenciers canadiens.

Le Sous-comité déplore tout d'abord l'existence de lacunes systémiques sur plan de l'élaboration des politiques. Les normes, vagues à l'extrême, sont inscrites dans des directives qui ne sont ni exécutoires 
ni accessibles au public et l'ensemble des procédures sont inéquitables. Dans son rapport, il recommande l'acceptation du principe voulant que la règle de droit prévale à l'intérieur des murs des prisons canadiennes et que l'arbitraire soit remplacé par des règles claires et publiques, des mesures disciplinaires équitables, des audiences dirigées par des présidents indépendants, ainsi que par des décisions justes et motivées (Canada, 1977b).

De façon plus générale, à cette époque, le Canada suit le mouvement international de reconnaissance des droits et libertés de la personne: en 1975, il endosse l'Ensemble des règles minima pour le traitement des détenus et, l'année suivante, il ratifie le Pacte international relatif aux droits civils et politiques (ONU, 1976a) de même que le Pacte international relatif aux droits économiques, sociaux et culturels (ONU, 1976b).

Cependant, la reconnaissance de principe à l'effet que les détenus conservent des droits pendant leur incarcération ne se transposait pas dans le réalité carcérale. Il faut dire que l'impopularité de la cause des droits des détenus n'offre pas un grand appui à la réforme législative. Améliorer la prison est rarement une priorité politique. Les gouvernements sont naturellement sensibles à l'opinion publique qui, historiquement, réclame souvent une augmentation de la sécurité, de la répression et la construction de nouvelles prisons. De plus, les autorités carcérales opposaient une résistance farouche au lobby en faveur des droits des détenus et à tout affaiblissement de leur pouvoir discrétionnaire. Les détenus se sont donc tournés vers les tribunaux pour faire reconnaître leurs droits et faire sanctionner le respect de l'équité et des principes de justice fondamentale par les autorités carcérales.

\section{Le passage par le droit et le rôle des tribunaux}

Les détenus se sont principalement battus contre l'arbitraire et la politique du secret qui prévalaient dans le monde fermé de l'incarcération. Ils ont réclamé le droit à des décisions équitables et le droit de se faire entendre tant sur le plan des audiences disciplinaires, du placement en isolement cellulaire, du transfèrement vers des institutions à sécurité plus élevée que sur le plan de l'obtention et de la perte d'une forme de libération conditionnelle.

Pendant longtemps, les tribunaux ont refusé d'assumer un rôle de surveillance des décisions internes du monde carcéral et ont adopté une attitude de laisser-faire (bands-off) en faisant la sourde oreille aux 
demandes des prisonniers. Divers arguments étaient invoqués pour justifier cette attitude: un trop grand nombre de poursuites saperait l'autorité, augmenterait les affrontements entre détenus et gardiens et créerait une instabilité malsaine. Les juges invoquaient également la déférence due à l'égard des autorités carcérales, lesquelles doivent jouir d'une large marge de manœuvre dans l'exécution de leur tâche difficile. Mais le principal motif ayant freiné l'intervention judiciaire dans le monde fermé de l'incarcération repose sur la conception que les personnes condamnées à l'emprisonnement perdent tous leurs droits et ne jouissent que de certains privilèges accordés de façon discrétionnaire.

Certaines contestations commencent malgré tout à porter leurs fruits. En 1976, les prisonniers de l'unité spéciale de correction du pénitencier de la Colombie-Britannique obtiennent un jugement déclaratoire, dans l'affaire McCann, selon lequel leurs conditions de détention constituent une peine cruelle et inusitée au sens de la Déclaration canadienne des droits $^{2}$. (Canada, arrêt McCann, 1976). Toutefois, tant dans cette affaire que dans les quelques autres entendues au cours de cette période, les tribunaux refusent de reconnaître que les décisions des autorités carcérales affectent des «droits» et qu'elles sont soumises à la révision judiciaire (Lemonde, 1990). Selon la conception dominante prévalant à l'époque, les personnes incarcérées sont déchues de tout droit et, en conséquence, ne peuvent avoir aucune prétention d'ordre juridique susceptible de sanction judiciaire.

Sur ce point toutefois, un vent de changement commence à souffler et certains juges enregistrent des dissidences virulentes. Ainsi, le juge en chef Laskin de la Cour suprême du Canada s'est-il indigné en 1976 dans l'affaire Mitchell de la prétention des autorités carcérales qui réclament, selon lui, une immunité quasi totale dans l'exercice de leurs pouvoirs. Parlant de la Commission nationale des libérations conditionnelles, il s'exprime ainsi:

Le fait brutal est que la Commission revendique un pouvoir tyrannique qui est, à mon avis, sans précédent parmi les organismes administratifs habilités à statuer sur la liberté individuelle. Elle réclame un pouvoir absolu sur la personne d'un détenu, presque comme s'il s'agissait d'une simple marionnette au bout d'une ficelle. (Canada, arrêt Mitchell, 1976: 577)

Les tribunaux abandonnent peu à peu le principe voulant que les détenus perdent tous leurs droits à l'exception de ceux qui leur sont

2. Cour suprême du Canada, citée par la suite comme SC, 1960, c. 44. 
spécifiquement reconnus par la loi au profit du principe contraire à l'effet que les personnes incarcérées conservent tous leurs droits à l'exception de ceux qui leur sont expressément retirés par la loi. La plus haute cour du pays reprend ce principe à son compte, en 1979, dans l'arrêt Solosky.

En 1980, la Cour suprême affirme que «la règle de droit doit prévaloir à l'intérieur des murs des pénitenciers» et reconnaît pour la première fois que, malgré l'absence de dispositions législatives à cet effet, les tribunaux disciplinaires des pénitenciers sont soumis à une obligation d'agir équitablement et que le non-respect de cette obligation donne ouverture à la révision judiciaire (arrêt Martineau, 1980: 622). Les prisonniers ont droit à la même qualité de justice que les autres administrés.

Quelques années plus tard, soit en 1985, un nouveau pas est franchi par les tribunaux. Dans la trilogie Miller, Cardinal et Morin, la Cour suprême affirme que la réalité moderne de l'incarcération implique des variations substantielles de degré de liberté résiduelle; ainsi l'incarcération en ségrégation comporte une privation significative de liberté par rapport aux conditions de détention dans la population générale de la prison. Il s'agit en fait, dit la Cour, d'une nouvelle détention qui doit avoir son propre fondement juridique. Puisqu'il y a privation additionnelle de liberté, il est possible de contester la légalité de la décision ordonnant cette nouvelle détention. Les détenus peuvent donc recourir à l'babeas corpus pour sortir de la "prison dans la prison »" De la même façon, il est aujourd'hui possible de contester la révocation d'une libération conditionnelle puisque la réincarcération qui s'ensuit constitue une privation de liberté (Dumas, 1986).

Le changement de perspective a été total et les tribunaux se sont immiscés dans le silence de la loi pour y inclure des principes d'équité procédurale et les valeurs issues du code juridique culturel en vigueur dans la société globale. Ils ont créé ainsi des normes et des standards de justice carcérale. L'historique de la législation canadienne dans le domaine correctionnel démontre que l'émergence des droits des détenus et l'intégration de normes d'équité n'ont pas été le fruit de la volonté du législateur ou d'une concession de la part de l'administration (Lemonde, 1995a; 1995c). Le gouvernement s'est opposé de toutes les 
façons possibles à ce que les questions concernant les droits des détenus soient débattues devant les tribunaux. Le développement jurisprudentiel en droit carcéral s'est fait à son corps défendant et en dépit de tous les efforts qu'il a déployés pour contrecarrer l'émergence de nouvelles normes d'équité et d'un modèle légal plus formel ${ }^{4}$.

En ouvrant la porte d'un forum où les plaintes des prisonniers pouvaient être entendues, les tribunaux ont contribué à réduire l'écart entre les personnes incarcérées et les autres catégories d'administrés. Par la suite, l'entrée en vigueur de la Charte canadienne des droits et libertés ${ }^{5}$ en 1982 et les ordonnances judiciaires consacrant l'obligation d'agir équitablement ont entraîné l'application d'impératifs concrets de justice comme le droit à une décision non secrète et équitable, comprenant le droit de connaître la preuve contre soi et de répondre, l'accès au dossier, le droit d'être présent aux auditions, le droit à une décision motivée et le droit à la représentation par avocat devant certaines instances décisionnelles.

Contraintes par les nombreuses ordonnances judiciaires rendues pendant les années 1980, les diverses autorités législatives et administratives commencent timidement et de façon progressive à intégrer de nouveaux standards de justice et d'équité. Le règlement et les directives sont amendés à plusieurs reprises pour codifier diverses facettes du discours des droits fondamentaux et de l'obligation d'agir équitablement.

La nouvelle Loi sur le système correctionnel et la mise en liberté sous condition $^{6}$, en vigueur depuis novembre 1992, se démarque radicalement du type de législations ayant prévalu au cours des cent cinquante années précédentes en ce qu'elle intègre et consacre les notions de dignité humaine et de droits fondamentaux des personnes incarcérées.

Les énoncés de principe visent l'accroissement de la transparence et le respect des droits des détenus. L'un d'eux est la codification de la norme établie par la Cour suprême que «le détenu continue à jouir des droits et privilèges reconnus à tout citoyen, sauf ceux dont la suppression ou restriction est une conséquence nécessaire de la peine infligée» (SC, 1992, с 20).

4. Pour une analyse de la résistance des autorités exécutives et carcérales à ce développement, voir Lemonde (1995c).

5. Loi constitutionnelle de 1982, annexe B de la Loi de 1982 sur le Canada (1982, R.-U., c. 11).

6. S.C. 1992, c. 20. Le Québec a aussi suivi le mouvement en amendant son Règlement sur les établissements de détention. S-4.01, r.1, pour y inclure plusieurs garanties procédurales. 
Un autre principe voulant que les décisions du service soient claires et équitables est également une codification de principes jurisprudentiels bien établis. Le prochain principe est le résultat du discours des droits généralement admis dans la société. On prévoit ainsi que les programmes et les méthodes doivent respecter les différences ethniques, culturelles et linguistiques ainsi qu'entre les sexes, et tenir compte des besoins propres aux femmes, aux Autochtones et autres groupes particuliers.

De nombreuses dispositions consacrent les droits fondamentaux des personnes incarcérées. La plupart des articles concernant les mesures disciplinaires, les transfèrements, l'isolement préventif ou punitif, les conditions d'incarcération, les fouilles, sont des intégrations par le législateur des règles jurisprudentielles concernant l'application des principes de justice fondamentale en milieu carcéral. Ainsi, le détenu doit pouvoir avoir recours à l'assistance d'un avocat lorsqu'il est placé en isolement ou lorsqu'il fait l'objet d'un projet de transfèrement. Il doit également avoir accès aux publications juridiques.

Les droits énumérés sont nombreux: droit à un milieu sain, et exempt de pratiques visant à détériorer la dignité humaine, droit à des soins de santé satisfaisant aux normes professionnelles reconnues, libre accès à la procédure de grief sans crainte de représailles. Il est interdit d'user de moyens de contrainte à titre de sanction, de faire subir un traitement inhumain, cruel ou dégradant, de procéder à un traitement médical expérimental sans avoir obtenu le consentement libre et éclairé, ou d'ordonner l'alimentation forcée d'un gréviste de la faim.

Le détenu jouit de la liberté d'association et de la liberté de religion. Il a le droit d'entretenir des relations avec sa famille et ses amis, notamment par des visites et de la correspondance. Finalement, les prisonniers ont le droit de participer aux décisions concernant la population carcérale, à l'exception des questions de sécurité. La reconnaissance de ce droit est très significative, car comme l'explique la professeure Jackman, nier aux personnes le droit de participer aux décisions les affectant a un impact sur leur bien-être et génère un sens de «powerlessness, insignificance and isolation» (Jackman, 1990: 371).

Un autre exemple de l'importance de l'activisme judiciaire dans la reconnaissance de droits pour les personnes incarcérées est la question du droit de vote. La loi électorale fédérale et plusieurs lois électorales provinciales, à l'exception de la loi québécoise, prévoyaient expressément que les condamnés n'avaient pas le droit de vote pendant leur incarcération. À la demande de la Commission royale d'enquête sur la 
réforme électorale, Pierre Landreville et moi-même avons procédé à une étude sur la question du droit de vote des détenus et avons recommandé l'abrogation de cette exclusion (Landreville et Lemonde, 1991). La Commission a plutôt recommandé que l'exclusion ne frappe que les personnes reconnues coupables d'un crime pouvant entraîner l'emprisonnement à vie et qui ont été condamnées à dix ans ou plus. Le gouvernement d'alors n'a pas suivi cette recommandation et a amendé la loi en spécifiant que sont exclues de la qualité d'électeur, les personnes purgeant une peine de deux ans ou plus ${ }^{7}$. C'est après de longues années de lutte devant les tribunaux que la Cour suprême invalidera, en 2002, cette exclusion jugée sans fondement et, par conséquent, inconstitutionnelle (Canada, arrêt Sauvé, 2002).

\section{La population carcérale}

Aux États-Unis, durant les années 1960, le mouvement abolitionniste prônait les peines de substitution, l'emprisonnement devant être réservé aux seuls «délinquants dangereux», les "dangerous few», soit 10 à $15 \%$ des contrevenants (Morris, 1974). Le second postulat de la recherche de Landreville en 1973, soit que l'emprisonnement ne se justifie que pour les délinquants qui mettent très sérieusement en danger la collectivité, s'inscrit dans la lignée de ce mouvement.

Reconnaissant que l'emprisonnement ne permet pas d'atteindre les objectifs de la peine, soit la protection de la société, la dissuasion et la réhabilitation, le principe de l'emprisonnement comme dernier recours a été repris dans le discours canadien. Plusieurs commissions ou groupes de travail mis sur pied depuis des décennies concluent que l'incarcération doit être utilisée avec retenue et être réservée à ceux pour qui elle est essentielle, c'est-à-dire ceux qui ont commis un crime grave ou violent et qui représentent un danger pour la société. Déjà, en 1976, le rapport du Comité canadien de la réforme pénale et correctionnelle, intitulé Justice pénale et correction : un lien à forger (Canada, 1969), recommandait d'éviter l'incarcération autant que possible et de n'utiliser cette peine que pour les infractions les plus graves.

En 1987, la Commission canadienne pour la détermination de la peine tire les conclusions suivantes: 1) aucune étude empirique ne permet d'affirmer que le mécanisme de sanction a un effet dissuasif;

7. Loi électorale du Canada, 1999, chap. 18, art. 23. 
2) bien qu'il soit évident qu'un détenu ne peut pas commettre la même infraction une fois qu'il est sous les verrous, la neutralisation d'un délinquant ne doit pas être envisagée comme un moyen de protection du public; 3) aucune donnée ne permet de dire que l'emprisonnement assure la réadaptation (Canada, 1987).

En 1988, le Comité permanent de la Justice et du Solliciteur général écrit dans Des responsabilités à assumer:

Concrètement, le recours à l'emprisonnement comme principale punition pour toutes sortes d'infractions à la loi n'est pas une approche défendable. La plupart des délinquants ne sont ni violents ni dangereux. Il est peu probable que leur comportement s'améliore par suite d'un séjour en prison. (Canada, 1988: 81)

C'est dans la foulée de ces constatations sur l'échec de la prison, que le législateur canadien a, en 1995, adopté le projet de loi C-41 sur la détermination de la peine, qui codifie l'idée que l'emprisonnement ne doit être imposé que dans les cas les plus graves.

Malgré ces déclarations de principe, c'est exactement l'inverse qui s'est passé, dans une mesure moindre que chez nos voisins du sud, mais d'une manière tout aussi alarmante chez nous. Plusieurs initiatives législatives des dernières années sont destinées à emprisonner plus et pour plus longtemps.

En 1999, dans l'affaire Gladue, la Cour suprême constatait que:

Le Canada fait figure de chef de file mondial dans de nombreux domaines, et particulièrement en matière de politiques sociales progressistes et de droits de la personne. Malheureusement, notre pays se distingue aussi, à l'échelle mondiale, par le nombre de personnes qu'il met en prison. Bien que les États-Unis, avec plus de 600 détenus pour 100000 habitants, aient de loin le plus haut taux d'incarcération parmi les démocraties industrialisées, le taux au Canada est d'environ 115 à 130 détenus pour 100000 habitants, ce qui le place au deuxième ou au troisième rang. Ces statistiques relatives au taux d'incarcération n'inspirent aucune fierté. (Canada, arrêt Gladue, 1999: par. 52)

Selon les données du Solliciteur général, de 1989 à 1995, la population carcérale dans les pénitenciers canadiens s'est accrue de $22 \%$ et, dans les prisons provinciales, de $12 \%$ (Canada, 1996c). Si le nombre de délinquants sous responsabilité fédérale a diminué entre 1996 et 2002, il n'en demeure pas moins que le taux d'incarcération au Canada était de 130 pour 100000 habitants en 2005 (Canada, 2006). Chez les 
Autochtones, ce taux s'élève à 1024 pour 100000 adultes. Ceux-ci constituent $2,7 \%$ de la population canadienne adulte, alors qu'ils représentent environ $18,5 \%$ des détenus sous responsabilité fédérale (Canada, 2006).

Ce taux d'incarcération est plus élevé que dans plusieurs pays de l'Europe de l'Ouest comme l'Allemagne (95), la France (85), la Finlande (70), la Suisse (68) et le Danemark (64), mais moins élevé qu'en Angleterre et au Pays de Galles (139) ou qu'aux États-Unis (702) (Canada, 2004). Selon Ivan Zinger:

Canada's incarceration rate continues to rank among the highest in the industrialized world. Many of our penitentiaries are full beyond capacity. The majority of our prison population is drawn from the ranks of the economically and socially disadvantaged; a disproportionate number of minorities, including Aboriginal persons, are locked up in our prisons. (Zinger, 1998: 6)

L'augmentation de la population carcérale n'est pas due à l'augmentation de la criminalité violente mais au durcissement des politiques pénales. Malgré une diminution du taux de criminalité au cours des dernières années, un plus grand nombre de délinquants sont condamnés à des peines d'emprisonnement et ces peines sont plus longues; dans les établissements fédéraux, on accorde moins de mises en liberté sous condition et les révocations sont plus fréquentes (Canada, 2004). Il faut ajouter l'augmentation importante des infractions pour lesquelles sont prévues des sentences minimales.

On a encore trop souvent recours au système de justice pénale pour tenter de régler des problèmes sociaux, alors que, bien souvent, des mesures moins draconiennes conviendraient davantage. Loïc Wacquant a longuement expliqué que l'exaltation de la loi et de l'ordre et la lutte contre l'insécurité ont débouché sur un durcissement des sanctions pénales. Celles-ci, de manière disproportionnée, frappent surtout les exclus et les marginalisés. D'un filet de protection sociale, nous sommes passés à un filet policier et pénal (Wacquant, 1999).

Cette «criminalisation de la misère » ou «gestion sociale par le droit pénal» (Robert, 1998) en Amérique du Nord n'est pas nouvelle comme l'ont démontré Rothman (1971) ou, plus près de nous, Fecteau (1985; 1989 ) et Laplante (1984). Elle prend des formes discriminatoires: les Noirs aux États-Unis, les Autochtones au Canada (Canada, 1996b; Jackson, 1988). Selon Statistique Canada, les personnes les plus sus- 
ceptibles d'être incarcérées sont les jeunes hommes, pauvres et autochtones. De plus, ils sont moins instruits et plus susceptibles d'être au chômage au moment de leur incarcération (Canada, 1996d). Cette situation était la même en 2005 (Canada, 2005).

Pierre Landreville s'est aussi intéressé à la question de la criminalisation de la misère. Avec Danièle Laberge, il a étudié les fonctions de la prison sur les exclus, en particulier, les itinérants (Landreville, Laberge et al., 1998). Les chercheurs expliquent que l'infraction pénale est une construction juridico-politique. Ce sont les législateurs qui décident de ce qui est "pénalisable», puis les agents de l'appareil pénal, policiers, procureurs, juges, décideront quel comportement doit être puni. Ils en concluent que:

Cette construction de la «réalité pénale» s'opère toujours dans un contexte de rapports de pouvoir. Il n'est donc pas surprenant de constater que ce sont surtout les comportements de ceux qui sont les plus vulnérables qui sont criminalisés et que ce sont les «sans pouvoir» qui sont le plus souvent définis comme délinquants et éventuellement incarcérés. (Barak et Bohm,1989: 155)

Une étude menée par Cécile Bellot et d'autres, du Centre international de criminologie comparée, La judiciarisation des populations itinérantes de 1994 à 2004 (Bellot, Raffestin et al., 2005), montre que les constats d'infraction émis à l'encontre des personnes fréquentant la rue a quadruplé au cours de ces années, que le recours à l'emprisonnement pour non-paiement d'amende est généralisé pour cette catégorie de personnes, que les coûts sociaux et judiciaires liés à cette problématique ont explosé et, finalement, qu'il y a surjudiciarisation de certaines populations itinérantes, notamment les jeunes.

Il apparaît donc que non seulement l'incarcération ne diminue pas mais aussi qu'elle opère de façon discriminatoire et a une fonction d'exclusion des populations les plus marginalisées et les plus vulnérables de notre société. Ce fait a d'ailleurs été reconnu à l'échelle planétaire par les Nations Unies. En effet, de nombreux rapports démontrent qu'après le type de crime commis, les deux facteurs les plus déterminants dans l'imposition d'une sentence lourde sont la classe sociale et l'origine ethnique (ONU, 1998; 1999).

$\mathrm{Au}$ Canada, les nouveaux principes de détermination de la peine voulant que la privation de liberté ne soit envisagée qu'en dernier recours et que des peines substitutives soient examinées, principalement 
pour les délinquants autochtones, vont dans le sens de diminuer la population carcérale. Mais ces peines substitutives sont continuellement remises en question. Ainsi, le gouvernement actuel entend réduire énormément l'application de la peine avec sursis ${ }^{8}$.

\section{Discussion}

Il apparaît donc clairement que le mouvement des droits des détenus a eu un impact sur l'émergence de nouvelles normes respectant la dignité humaine et les droits fondamentaux des personnes incarcérées, comme le souhaitait Landreville. Cependant, il faudrait encore en questionner l'impact réel sur les pratiques carcérales, les conditions de détention et les mentalités des acteurs concernés. Entre l'acceptation de principe par les branches législative et judiciaire de la règle de droit et sa translation dans la pratique quotidienne, il y a loin de la coupe aux lèvres. Les très graves incidents survenus en avril 1994 au pénitencier pour femmes à Kingston sont symptomatiques. À la suite d'un reportage extrêmement choquant montrant des détenues toutes nues, fouillées et arrosées par des membres d'un groupe d'intervention tactique composé uniquement d'hommes, démonstration de force abusive et dégradante, le gouvernement a créé une commission d'enquête, présidée par l'honorable juge Louise Arbour (Canada, 1996a). Celle-ci a infligé un sérieux blâme au Service correctionnel et à certains de ses membres et émis plusieurs recommandations.

Le fait que le nouveau système soit plus formel et rationnel ne signifie pas automatiquement qu'il soit plus juste. Pour le professeur Jackson, qui a mené une nouvelle recherche sur la qualité de la prise de décisions à l'intérieur des murs et le respect des droits des détenus trente ans après la parution de son premier article, il y a encore beaucoup à faire. Mises à part les décisions disciplinaires prises par des présidents indépendants, toutes les autres décisions affectant les droits et libertés des détenus, comme la ségrégation administrative, les transfèrements et les visites, sont prises par des employés du système. Il recommande en conséquence que ces décisions affectant les droits soient prises par des personnes indépendantes (Jackson, 2002).

8. Projet de loi C-9, Loi modifiant le Code criminel (emprisonnement avec sursis), 2006, $39^{\mathrm{e}}$ législature, $1^{\mathrm{re}}$ session. 
Si de graves problèmes persistent et si des îlots de résistance à la réforme sont encore perceptibles, la rationalisation et la «légalisation» des pratiques décisionnelles ont contribué de façon certaine à la réduction de l'arbitraire, à la transparence et à la limitation de l'autonomie institutionnelle dans le maintien de l'ordre et le contrôle disciplinaire des détenus. Il est clair que le premier postulat de Landreville, énoncé en 1973, s'est réalisé, à savoir que les droits des détenus sont aujourd'hui reconnus et inscrits dans la loi. Comme il l'a lui-même écrit:

Même si certains ont mis l'accent sur les limites du juridisme et soutiennent que «sur le plan humain, il convient de parler davantage de régression ${ }^{9}$ », on peut néanmoins constater que cette reconnaissance a eu des conséquences sur les rapports de pouvoir dans les établissements, sur le fonctionnement quotidien de ces institutions. Les règles du jeu ont changé. Les prises de décision sont moins discrétionnaires et doivent respecter certaines garanties procédurales. (Lemonde et Landreville, 2002: 87)

Les droits civils et les garanties procédurales, de même que les droits économiques, sociaux et culturels des détenus sont législativement reconnus au Canada, mais leur mise en œuvre demeurera illusoire tant que la tendance actuelle d'augmentation de la longueur des sentences et, partant, de la population carcérale, prévaudra.

Le professeur Jackson est plutôt pessimiste sur l'avenir du mouvement des droits des détenu(e)s quand il écrit:

Disturbingly, as the issue of prisoners' rights has emerged from the legal shadows onto a clearly defined landscape, it appears dangerously close to being eclipsed by rising concern for victims of crime and a growing fear about the erosion of public safety (a fear strangely impervious to the decrease in the rate of violent crime). (Jackson, 2002)

Le travail de pionnier de Pierre Landreville en matière des droits des détenu(e)s a eu un impact majeur sur l'évolution des mentalités et des politiques pénales en la matière. Sa pensée doit survivre malgré le retour du balancier et la pression pour «adopter des systèmes carcéraux plus austères (sans superflu), imposer de nouvelles restrictions aux détenus et renforcer les exigences en matière de sécurité» (Zinger, 2006:141).

Garantir les droits des détenu(e)s continue d'être un défi majeur pour tous les pays et pour le Canada en particulier. En ce sens, la Canada

9. Lemire (1991). Vingt ans de droits des détenus au Québec. Criminologie, XXIX (1), 74. 
devrait ratifier le Protocole facultatif se rapportant à la Convention contre la torture et autres peines ou traitements cruels, inbumains ou dégradants qui instaure un mécanisme externe de surveillance des lieux de détention, à l'image du Comité européen de prévention de la torture. Landreville a d'ailleurs plaidé en ce sens dans un article récent (Lehalle, Landreville et al., 2006). Pour lui et ses collègues, dans l'actuel climat d'inquiétude face à la menace terroriste, et j'ajouterais dans l'actuel climat d'insécurité, réelle ou entretenue, de même que dans cette ère de la gestion du risque actuariel, «un mécanisme international de contrôle sur le modèle du Comité européen apparaît plus que souhaitable, même nécessaire».

Dans cet article, son dernier à ma connaissance sur la question des droits des détenu(e)s, Landreville réaffirme son engagement envers le droit international des droits de la personne. Quand, en 1973, il plaidait en faveur de l'application au Canada de l'Ensemble des règles minima pour le traitement des détenus (Landreville, 1973), Landreville était un véritable précurseur, voire un visionnaire. À cette époque, se référer aux normes internationales de protection des droits humains était rarissime tant chez les politologues que chez les juristes. En ce sens, il a influencé plusieurs générations d'universitaires, de décideurs et de défenseurs des droits, et a contribué non seulement au mouvement pour la reconnaissance des droits des personnes incarcérées au Québec et au Canada mais également à l'internationalisation et à l'irréversibilité de ce mouvement.

\section{Références}

Bellot, C., Raffestin, I., Royer, M.-N., \& Noël, V. (2006). La judiciarisation des populations itinérantes de 1994 à 2004. Rapport de recherche non publié.

Berkamn, R. (1979). Opening the Gates: The Rise of the Prisoner's Movement. Lexington: Health \& Company.

Bronstein, A. J. (1980). Prisoners' Rights : A History. In G.P. Alpert (Ed.), Legal Rights of Prisoners. London: Sage Publications.

Canada (1969). Justice pénale et correction: un lien à forger. Comité canadien de la réforme pénale et correctionnelle. Ottawa: Imprimeur de la Reine.

Canada (1977a). Le rôle des services correctionnels fédéraux au Canada. Ottawa: Ministre des Approvisionnements et Services Canada.

Canada (1977b). Le Sous-comité sur le régime des institutions pénitentiaires au Canada. Comité permanent de la justice et des questions juridiques (rapport MacGuigan). Ottawa: Ministre des Approvisionnements et Services Canada.

Canada (1987). Réformer la sentence: une approche canadienne. Commission canadienne sur la détermination de la peine. Ottawa: Ministre des Approvisionnements et Services Canada. 
Canada (1988). Des responsabilités à assumer. Comité permanent de la Justice et du Solliciteur général. Rapport du Comité permanent de la Justice et du Solliciteur général sur la détermination de la peine, la mise en liberté sous condition et d'autres aspects du système correctionnel. Ottawa: Chambre des communes.

Canada. (1996a). Commission d'enquête sur certains événements survenus à la prison des femmes de Kingston. Ottawa: Ministre des Approvisionnements et Services Canada.

Canada (1996b). Par-delà les divisions culturelles: Rapport sur les autochtones et la justice pénale au Canada. Commission royale sur les peuples autochtones. La Commission, 1996; Canada. Commission royale sur les peuples autochtones. Rapport de la Commission royale sur les peuples autochtones, vol. 4, Perspectives et réalités. Ottawa: La Commission.

Canada (1996c). Croissance de la population carcérale. Rapport à l'intention des ministres responsables de la Justice du gouvernement fédéral, des provinces et des territoires. Ottawa: Ministère du Solliciteur général, mai 1996.

Canada (1996d). Recensement effectué dans les établissements correctionnels le 5 octobre 1996. Statistique Canada.

Canada (2004). Aperçu statistique: le système correctionnel et la mise en liberté sous condition. Ottawa: Sécurité publique et Protection civile Canada, décembre 2004.

Canada. (2006). Rapport annuel du Bureau de l'Enquêteur correctionnel, 20052006.

Cornil, P. (1968). Normes internationales pour le traitement des délinquants. Revue internationale de politique criminelle, 26 (3), 16.

Fecteau, J.-M. (1985). Régulation sociale et répression de la déviance au BasCanada au tournant du $19^{\mathrm{e}}$ siècle (1791-1815) Revue d'bistoire de l'Amérique française, 38, 499-521.

Fecteau, J.-M. (1989). Un nouvel ordre des choses: la pauvreté, le crime, l'État au Québec, de la fin du XVIII siècle à 1840. Montréal: VLB.

Feely, M., \& Hanson, R. (1990). The Impact of Judicial Intervention on Prisons and Jails: A Framework for Analysis and a Review of Literature. In J. Dilulio (Ed), Courts, Corrections and the Constitution. The Impact of Judicial Intervention on Prisons and Jails (12-49). New York: Oxford University Press.

Huff, R. (1980). The Discovery of Prisoners' Rights: A Sociogical Analysis. In G. P. Alpert (Ed), Legal Rights of Prisoners. London: Sage Publications.

Jackman, M. (1990). Case Comments. Fundamental Interests and Fundamental Justice. - The Right to Participate in Decision-Making in Canadian Prisons: Piché c. S.-G. of Canada. University of British Columbia Law Rev, 24, 361-379.

Jackson M. (1988). Locking up Natives in Canada. University of British Columbia Law Rev, 23, 215.

Jackson, M. (1974). Justice Behind the Walls - A Study of the Disciplinary Process in Canadian Penitentiary. Osgoode Hall Law Journal, 12 (1), 1-103.

Jackson, M. (2002). Justice Behind the Walls. Human Rights in Canada Prisons. Douglas \& McIntyre. En ligne: http://www.justicebehindthewalls.net 
Jacobs, J. (1983). The Prisoner's Rights Movement and Its Impacts. In New Perspectives on Prison and Imprisonment. Ithaca: Cornell University Press.

Kaiser, G. E. (1971). The Inmate as a Citizen: Imprisonment and the Loss of Civil Rights in Canada. Queen's Law Journal, 2, 208-277.

Landreville P., \& Dumont, H. (1974). Discipline et droits des détenus dans les institutions pénales au Québec. Revue canadienne de criminologie, 15, 412434.

Landreville, P. (1973). Lapplication des règles minima pour le traitement des détenus au Canada. Acta Criminologica, VI, 147-199.

Landreville, P., Laberge, D., Morin, D., \& Casavant, L. (1998). Logique d'action et fonctions de la prison. L'exclusion des itinérants par le droit pénal. In P. Robert (Dir), Actes de la 8e Journée en droit social et du travail, La gestion sociale par le droit pénal. La discipline du travail et la punition des pauvres (153-171). Cowansville: Les Éditions Yvon Blais.

Landreville, P., \& Lemonde, L. (1991). Le droit de vote des personnes incarcérées. In M. Cassisy (Dir), Les droits démocratiques et la réforme électorale au Canada (vol. 10, 31-111). Collection d'études de la Commission royale d'enquête sur la réforme électorale et le financement des partis. Toronto, Montréal: Dunburn Press, Wilson et Lafleur.

Landreville, P., Gagnon, A., \& Desrosiers, S. (1976). Les prisons de par ici. Montréal: Éditions Parti Pris.

Laplante, J. (1984). Prison et ordre social au Québec. Ottawa: Presses de l'Université d'Ottawa.

Lehalle, S., Landreville, P., \& Céré, J.-P. (2006). Le Comité européen de prévention de la torture: Mécanisme de contrôle des établissements de détention. Canadian Journal of Criminology and Criminal Justice, 48 (2), 223-250.

Lemonde, L. (1990). L’habeas corpus en droit carcéral. Cowansville: Les éditions Yvon Blais.

Lemonde, L. (1995a). Historique des normes juridiques dans les pénitenciers au Canada. Revue Criminologie, XXVIII (1), 97-117.

Lemonde, L. (1995b). L'évolution des normes dans l'institution carcérale. Revue canadienne Droit et Société/Canadian Journal of Law and Society, 10 (1), 125170.

Lemonde, L. (1995c). L'impact de l'intervention judiciaire sur l'évolution des normes canadiennes en matière de libération conditionnelle. Revue de droit de McGill, 40, 581- 620 .

Lemonde L., \& Landreville, P. (2002). La reconnaissance des droits fondamentaux des personnes incarcérées: l'expérience canadienne. In O. De Schutter \& Dan Kaminski (Dir), L'institution du droit pénitentiaire. Enjeux de la reconnaissance de droits aux détenus (69-88). Paris: Bruylant, L.D.G.D.

Morris, N. (1974). The Future of Imprisonment. Chicago: The University of Chicago Press.

National Council on Crime and Delinquency (1972). A Model Act for the Protection of Rights of Prisoners. New-Jersey: Paramus, NCCD. 
ONU (1955). Ensemble de règles minima pour le traitement des détenus. Résolution du premier congrès des Nations Unies pour la prévention du crime et le traitement des délinquants, Genève, 30 août 1955.

ONU (1976a). Pacte international relatif aux droits civils et politiques. 999 RTNU 187.

ONU (1976b). Pacte international relatif aux droits économiques, sociaux et culturels. 993 RTNU 13.

ONU (1998). Question of the violation of buman rights and fundamental freedoms in any part of the world. Doc. NU: E/CN4/1998/68/Add.3.

ONU (1999). Civil and political rights, including questions of disappearances and summary executions. Doc. NU: E/CN4/1999.39.

ONU (2002). Doc. A/RES57/199, 18 décembre 2002 (entrée en vigueur: 22 juin 2006).

Pires, A. P. (1991). La réforme pénale et la réciprocité des droits. Criminologie, XXIV (1), 77-104.

Robert P. (1998). Actes de la 8 Journée en droit social et du travail. La gestion sociale par le droit pénal. La discipline du travail et la punition des pauvres. In P. Robert (Dir). Cowansville: Les Éditions Yvon Blais.

Rothman D. (1971). The Discovery of the Asylum: Social Order and Disorder in the New Republic. Boston: Little, Brown.

Wacquant, L. (1999). Les Prisons de la misère. Paris: Raisons d'agir. Éditions du Seuil.

Zinger, I. (1998). Human Rights for All. Let's Talk, 23 (4), 4.

Zinger, I. (2006). Respect des obligations relatives aux droits de la personne et rôle de la surveillance externe. Canadian Journal of criminology and criminal Justice, 48 (2), 141-155.

\section{Jurisprudence}

Canada (1976). McCann c. La Reine [1976] 1 CF 570.

Canada (1976). Mitchell c. La Reine [1976] 2 RCS 570.

Canada (1980) Solosky c. La Reine [1980] 1 RCS 823.

Canada (1980). Martineau c. Comité de discipline de l'établissement de Matsqui ( $\left.n^{\circ} 2\right)$, [1980] 1 RCS 602.

Canada (1985). Morin c. Comité national chargé des cas d'U.S.D. [1985] 2 RCS 662.

Canada (1985). R. c. Miller [1985] 2 RCS 613.

Canada (1985). Cardinal c. Directeur de l'Établissement Kent [1985] 2 RCS 643.

Canada (1986). Dumas c. Directeur du Centre de détention Leclerc [1986] 2 RCS 459.

Canada (1999). R. c. Gladue [1999] 1 RCS 688.

Canada (2002). Sauvé c. Canada (Directeur général des élections) [2002] 3 RCS 519. 